\title{
A Classification of Regular Embeddings of Graphs of Order a Product of Two Primes
}

\author{
SHAO-FEI DU \\ Department of Mathematics, Capital Normal University, Beijing 100037, People's Republic of China \\ JIN HO KWAK \\ jinkwak@postech.ac.kr \\ Combinatorial and Computational Mathematics Center, Pohang University of Science and Technology, \\ Pohang 790-784, Korea \\ ROMAN NEDELA \\ Institute of Mathematics and Informatics, Slovak Academy of Sciences, 97400 Banská Bystrica, Slovakia
}

Received August 30, 2001; Revised March 17, 2003; Accepted April 1, 2003

\begin{abstract}
In this paper, we classify the regular embeddings of arc-transitive simple graphs of order $p q$ for any two primes $p$ and $q$ (not necessarily distinct) into orientable surfaces. Our classification is obtained by direct analysis of the structure of arc-regular subgroups (with cyclic vertex-stabilizers) of the automorphism groups of such graphs. This work is independent of the classification of primitive permutation groups of degree $p$ or degree $p q$ for $p \neq q$ and it is also independent of the classification of the arc-transitive graphs of order $p q$ for $p \neq q$.
\end{abstract}

Keywords: regular map, regular embedding, genus, arc-transitive graph, permutation group

2000 Mathematics Subject Classification: Primary 05C10, 05C25; Secondly 20B25

\section{Introduction}

A (topological) map is a cellular decomposition of an orientable closed surface. A common way to describe maps is to view them as 2-cell embeddings of graphs. An automorphism of a map is an automorphism of the underlying graph which extends to an orientation preserving self-homeomorphism of the supporting surface. It is well-known that the automorphism group of a map acts semiregularly on the set of arcs of the underlying graph and in an extreme case, when the action is regular, the map itself is called regular. A regular embedding of a graph is a 2-cell embedding of a graph into a surface in a way that the associated map is regular. Regular maps have been studied in connection with various branches of mathematics including Riemann surfaces and algebraic curves. For more information about regular maps and their connections to other fields of mathematics we refer the reader to $[12-14,18,19$, $24,25]$.

One significant problem in the theory of regular maps is to classify the regular embeddings of a given underlying graph. Generally, this is very difficult. Regular embeddings of the complete graph $K_{n}$ are classified in $[1,11,26]$. In [5], the authors classified the regular 
embeddings of complete $n$-multipartite graphs $K_{p, p, \ldots, p}$, where $p$ is a prime and $n$ is a positive integer. It is known [7] that the automorphism group of a regular map is a twogenerator group acting with a cyclic vertex-stabilizer, and conversely, any two-generator group with one generator being involution determines a regular map. This regular map depends on the choice of the generators, and one can usually derive more than one regular map from such a group.

When classifying regular embeddings of graphs, a technique often used is to project a given map onto a smaller one, and then to employ information about the quotient. Although underlying graphs of regular maps may have multiple edges, a regular map with multiple edges projects onto another one with a simple underlying graph that has the same set of vertices and the same adjacency relation. Hence regular maps with multiple edges can be described as some "extensions" of regular embeddings of simple graphs. Therefore from now on all the maps considered throughout the paper will be assumed to have simple underlying graphs. Since the regular maps with $p$ or $p q$ vertices, where $p$ and $q$ are primes, appear as quotients of many other regular maps, they are suitable candidates to be dealt with. The classification of regular maps with $p$ vertices was achieved in [5]. The classification of such regular maps can be derived from the classification of arc-transitive graphs with prime number of vertices, see [3]. In this paper, we shall give a classification of regular embeddings of connected arc-transitive simple graphs of order $p q$, where $p$ and $q$ are primes (not necessarily distinct).

Since the classification of arc-transitive graphs of order $p q(p \neq q)$ is known (see [17-22]), it is possible to use these results for our purpose. However, there are some arguments that do not support this idea. First of all, many different families of graphs included in that classification have to be checked unnecessarily. For some of these families, either there are no regular maps, or the classification is highly non-trivial. This situation can be well demonstrated in the family of complete $q$-partite graphs $K_{p, \ldots, p}$ which are obviously arc-transitive of order $p q$. These graphs admit a regular embedding into a surface if and only if either $q \leq 3$, or $p=q$, see [5]. Perhaps the most important argument to attack the problem independently of the classification of arc-transitive graphs of order $p q$ uses the fact that it depends on the classification of primitive groups of degree $p q$ (see [15]). Since however the classification of primitive groups of degree $p q$ depends in turn on the classification of finite simple groups, it is worth classifying regular maps with $p q$ vertices independently of it. Our approach avoids the employment of this classification. In fact, it is independent of the classification of finite simple groups.

The paper is organized as follows. After this introductory section, a brief description of algebraic maps and some preliminary group theoretical results will be given in Sections 2 and 3. Section 4 contains the proof of the classification theorem (see Theorem 4.8). In Section 5 we compute the genera of the maps obtained in Theorem 4.8.

\section{Algebraic maps}

Let $\mathcal{G}=\mathcal{G}(V, D)$ be a simple graph with vertex set $V=V(\mathcal{G})$ and arc set $D=D(\mathcal{G})$. By $S_{V}$ and $S_{D}$ we denote the symmetric groups on the vertex set and on the arc set, respectively. The involution $L$ in $S_{D}$ interchanging the two arcs underlying every given edge is called the 
arc-reversing involution. An element $R$ in $S_{D}$ which cyclically permutes the arcs initiated at $v$ for each vertex $v \in V(\mathcal{G})$ is called a rotation. Since the graph $\mathcal{G}$ has been assumed to be simple, $\operatorname{Aut}(\mathcal{G})$ is considered as a subgroup of both $S_{V}$ and $S_{D}$, and the same notation is used for convenience. In the investigation of maps, it is often useful to replace topological maps on orientable surfaces with their combinatorial counterparts. It is well-known that graph embeddings into orientable surfaces can be described by means of rotations (see $[8,13])$. A map $\mathcal{M}$ with underlying graph $\mathcal{G}$ can be identified with a triple $\mathcal{M}=\mathcal{M}(\mathcal{G} ; R, L)$, where $R$ is a rotation and $L$ is the arc-reversing involution of $\mathcal{G}$. By the connectivity of $\mathcal{G}$, $\operatorname{Mon}(\mathcal{M}):=\langle R, L\rangle$ is a transitive subgroup of $S_{D}$. Given two maps $\mathcal{M}_{1}=\mathcal{M}\left(\mathcal{G}_{1} ; R_{1}, L_{1}\right)$ and $\mathcal{M}_{2}=\mathcal{M}\left(\mathcal{G}_{2} ; R_{2}, L_{2}\right)$, a graph isomorphism $\phi: \mathcal{G}_{1} \rightarrow \mathcal{G}_{2}$ is called a map isomorphism from $\mathcal{M}_{1}$ to $\mathcal{M}_{2}$ if $R_{1} \phi=\phi R_{2}$, noting that $L_{1} \phi=\phi L_{2}$ holds in any case. In particular, if $\mathcal{M}_{1}=\mathcal{M}_{2}=\mathcal{M}$, then $\phi$ is called an automorphism of $\mathcal{M}$. The automorphisms of $\mathcal{M}$ form a group $\operatorname{Aut}(\mathcal{M}) \leq \operatorname{Aut}(\mathcal{G})$, called the automorphism group of the map $\mathcal{M}$. By this definition, $\operatorname{Aut}(\mathcal{M}) \leq C_{S_{D}}\left(\operatorname{Mon}(\mathcal{M})\right.$ ), the centralizer of $\operatorname{Mon}(\mathcal{M})$ in $S_{D}$. Also $\operatorname{Aut}(\mathcal{M})$ acts semi-regularly on $D$, which follows from the transitivity of $\operatorname{Mon}(\mathcal{M})$ on $D$. If the action is regular, the map $\mathcal{M}$ is called regular. As a consequence of some well-known results in a permutation group theory (see [9, I.6.5]), we infer that in a regular map $\mathcal{M}$, the two associated permutation groups $\operatorname{Aut}(\mathcal{M})$ and $\operatorname{Mon}(\mathcal{M})$ on $D$ can be viewed as the right and the left regular representations of an abstract group $G$, so that $G \cong \operatorname{Aut}(\mathcal{M}) \cong \operatorname{Mon}(\mathcal{M})$, mutually centralizing each other in $S_{D}$ (see [13]).

It is also possible to describe a regular map in terms of its automorphism group, and its underlying graph as a coset graph. Let us first recall the definition of coset graphs (see [16] for example). Let $G$ be a finite group and $H$ a proper subgroup of $G$ with $\bigcap_{g \in G} H^{g}=1$. Let $B$ be a double coset of $H$ in $G$ such that $B=B^{-1}$. From now on, we use $\mathcal{G}=\mathcal{G}(G ; H, B)$ to denote the coset graph with $V(\mathcal{G})=\{H g \mid g \in G\}$ and $D(\mathcal{G})=\{(H g, H b g) \mid b \in$ $B, g \in G\}$. Note that $G$ acts faithfully and arc-transitively on the coset graph by right multiplication. In what follows, the group $G$ is often identified with the corresponding group of right multiplications.

Definition 2.1 Let $G=\langle r, \ell\rangle$ be a finite two-generator group with $\ell^{2}=1$ and $\langle r\rangle \cap\langle r\rangle^{\ell}=$ 1. By an algebraic map $\mathcal{M}(G ; r, \ell)=(\mathcal{G} ; R)$, we mean the map whose underlying graph is the coset graph $\mathcal{G}=\mathcal{G}(G ;\langle r\rangle,\langle r\rangle \ell\langle r\rangle)$ and rotation $R$ is determined by $e^{R}=e^{g_{1}^{-1} r g_{1}}=$ $\left(\langle r\rangle g_{1},\langle r\rangle g_{2} g_{1}^{-1} r g_{1}\right)$ for any arc $e=\left(\langle r\rangle g_{1},\langle r\rangle g_{2}\right)$ in $D(\mathcal{G})$.

By the definition given above, $G$ acts arc-regularly on $\mathcal{G}$ (by right multiplication) and preserves the rotation $R$ of the map $\mathcal{M}(G ; r, \ell)$. Therefore any algebraic map $\mathcal{M}$ is regular, with $\operatorname{Aut}(\mathcal{M}) \cong \operatorname{Mon}(\mathcal{M}) \cong G$. It is a matter of routine to check (see [13, 18]) that every regular embedding of a graph can be described by an algebraic map, and that two such algebraic maps $\mathcal{M}\left(G ; r_{1}, \ell_{1}\right)$ and $\mathcal{M}\left(G ; r_{2}, \ell_{2}\right)$ are isomorphic if and only if there exists an automorphism $\sigma \in \operatorname{Aut}(G)$ such that $r_{1}^{\sigma}=r_{2}$ and $\ell_{1}^{\sigma}=\ell_{2}$. Note that isomorphic regular maps have isomorphic automorphism groups. Therefore one can transfer the classification problem of regular embeddings of a given graph into a purely group theoretical problem. More precisely, one may classify all the regular maps with a given underlying graph $\mathcal{G}$ of valency $n$ in the following two steps: 
(1) Find the representatives $G$ (as abstract groups) of the isomorphism classes of arc-regular subgroups of $\operatorname{Aut}(\mathcal{G})$ with cyclic vertex-stabilizers.

(2) For each group $G$ given in (1), determine all the algebraic regular maps $\mathcal{M}(G ; r, \ell)$ with underlying graphs isomorphic to $\mathcal{G}$, or equivalently, determine the representatives of the orbits of $\operatorname{Aut}(G)$ on the set of generating pairs $(r, \ell)$ of $G$ such that $|r|=n$, $|\ell|=2$ and $\mathcal{G}(G ;\langle r\rangle,\langle r\rangle \ell\langle r\rangle) \cong \mathcal{G}$.

\section{Preliminary results in group theory}

In this section, some group theoretical results are given. Let's first introduce some notation.

$\mathrm{By}(r, s)$ and $[r, s]$, we denote the greatest common divisor and the least common multiple of two positive integers $r$ and $s$, respectively; and by $[a, b]$ (with an abuse of notation) the commutator of two elements $a$ and $b$ in a group. By $\mathbb{F}_{p}, \mathbb{Z}_{n}$ and $\mathbb{D}_{2 n}$, we denote the finite field of $p$ elements, the cyclic group of order $n$ and the dihedral group of order $2 n$, respectively. For a group $G$ and a subgroup $H$ of $G$, we use $G^{k+}$ to denote the subgroup $\langle g| g \in G, g^{k}=$ 1) of $G$, and use $C_{G}(H)$ and $N_{G}(H)$ to denote the centralizer and normalizer of $H$ in $G$, respectively. A semidirect product of the group $N$ by the group $H$ is denoted by $N: H$. For a ring $S$, let $S^{*}$ be the multiplicative group of $S$. Finally, by $V=V(2, p), \operatorname{PG}(V), \operatorname{AG}(V)$, $\operatorname{GL}(2, p), \operatorname{PGL}(2, p)$, and $\operatorname{AGL}(2, p)$, respectively, we denote the 2-dimensional row linear space, projective geometry, affine geometry, general linear group, projective general linear group, and affine transformation group over the field $\mathbb{F}_{p}$.

For any $\alpha \in V$, we denote by $t_{\alpha}$ the translation corresponding to $\alpha$ in $\operatorname{AG}(V)$ and by $T$ the translation subgroup of $\operatorname{AGL}(2, p)$. Then $\operatorname{AGL}(2, p) \cong T: \operatorname{GL}(2, p)$. We adopt matrix notation for GL(2,p) and, for convenience, denote a matrix $x=\left(a_{i j}\right)_{2 \times 2}$ by $x=\| a_{11}$, $a_{12} ; a_{21}, a_{22} \|$. We have $g^{-1} t_{\alpha} g=\left(t_{\alpha}\right)^{g}=t_{\alpha g}$ for any $t_{\alpha} \in T \leq \operatorname{AGL}(2, p)$ and any $g \in \operatorname{GL}(2, p) \leq \operatorname{AGL}(2, p)$. Hereafter, let us fix $a=t_{(1,0)}, b=t_{(0,1)}$, so that $\langle a, b\rangle=T$ is the translation subgroup of $\operatorname{AGL}(2, p)$. Let $G=T:\langle x\rangle$, where $x=\|e, u ; f, w\|$ is an element of order $n$ in GL(2,p). Then as an abstract group, $G$ can be presented by

$$
G=\left\langle a, b, x \mid a^{p}=b^{p}=x^{n}=[a, b]=1, a^{x}=a^{e} b^{u}, b^{x}=a^{f} b^{w}\right\rangle .
$$

Conversely, any group $G$ given by (3.1) can be viewed as a subgroup of $\operatorname{AGL}(2, p)$ containing $T$, by identifying $a, b, x$ with $t_{(1,0)}, t_{(0,1)}$ and $\|e, f ; u, w\|$, respectively. This identification will be frequently used later.

The knowledge of 2-dimensional linear groups as well as that of projective groups or of affine groups will be used throughout this paper, and the reader is assumed to be familiar with it. To make the proofs more transparent in what follows, we have decided to include some known facts. Proposition 3.1 can be extracted from [23] and [4], and Proposition 3.2 can be obtained from Proposition 3.1 and some results in $[9$, I.8].

Proposition 3.1 Let $p$ be an odd prime. Then the maximal subgroups of $\operatorname{PGL}(2, p)$ lie in the following: one conjugacy class of subgroups isomorphic to $\mathbb{Z}_{p}: \mathbb{Z}_{p-1}$; one class isomorphic to $\mathbb{D}_{2(p-1)}$, when $p \geq 7$; one class isomorphic to $\mathbb{D}_{2(p+1)}$; one class isomorphic to $S_{4}$, when either $p=5$ or $p \equiv 3,13,27,37(\bmod 40)$; and one subgroup isomorphic to $\operatorname{PSL}(2, p)$. 
Proposition 3.2 For a prime $p$, suppose $\theta$ is a primitive element of $\mathbb{F}_{p}$, so that $\mathbb{F}_{p}^{*}=\langle\theta\rangle$, and let $G=\mathrm{GL}(2, p), Z=Z(G)$ and $\bar{G}=\operatorname{PGL}(2, p)$. Then

(1) $\operatorname{GL}(2,2)=\langle x, y\rangle \cong \mathbb{D}_{6}$, where $x=\|1,1 ; 1,0\|$ and $y=\|0,1 ; 1,0\|$.

(2) For $p \geq 3$, all the elements of the form $\|e, f \theta ; f, e\|$ form a cyclic subgroup $H$ of $G$, which is of order $p^{2}-1$ and contains $Z$. Moreover, $N_{G}(H) \cong H:\langle y\rangle$, where $y=\|1,0 ; 0,-1\|$ is an involution. For any $n$ satisfying $n \mid\left(p^{2}-1\right)$ but $n \nmid(p-1)$, each cyclic subgroup of order $n$ is conjugate to a subgroup of $H$. Each irreducible subgroup $L$ of $\operatorname{GL}(2, p)$ on its action on $V$ is conjugate to a subgroup of $H$, and the action $\bar{L}:=L Z / Z$ on $\mathrm{PG}(V)$ is regular.

(3) For $p \geq 3$, let $D$ be the diagonal subgroup of $G$. Then $N_{G}(D)=D:\langle y\rangle$, where $y=\|0,1 ; 1,0\|$ is an involution. For any two divisors $n$ and $m$ of $p-1$, each subgroup isomorphic to $\mathbb{Z}_{n} \times \mathbb{Z}_{m}$ is conjugate to a subgroup of $D$. Moreover, for each element $h$ in $D \backslash Z, \bar{h}:=h Z / Z$ fixes precisely two points in $\mathrm{PG}(V)$.

(4) For $p \geq 3$, G contains one class of subgroups isomorphic to $\mathbb{Z}_{p}: \mathbb{Z}_{p-1}$, with a representative $H=\langle x, y\rangle$, where $x=\|1,1 ; 0,1\|$ and $y=\|1,0 ; 0, \theta\|$. Moreover, $\bar{H}:=H Z / Z$ is a point-stabilizer of $\operatorname{PGL}(2, p)$ in the action on $\operatorname{PG}(V)$.

Lemma 3.3 Let $F=T:\langle x\rangle$ and $F^{\prime}=T:\left\langle x^{\prime}\right\rangle$ be two subgroups of $A:=\operatorname{AGL}(2, p)$, where $T$ is the translation subgroup, and $x$ and $x^{\prime}$ are nontrivial elements in $G=\operatorname{GL}(2, p)$. Suppose $\sigma$ is an isomorphism from $F$ to $F^{\prime}$ mapping $\langle x\rangle$ to $\left\langle x^{\prime}\right\rangle$. Then there exists an element $u \in G$ such that $\sigma=\left.I(u)\right|_{F}$, where $I(u)$ is the inner automorphism of $A$ induced by $u$. In particular, if $\langle x\rangle=\left\langle x^{\prime}\right\rangle$ then $u \in N_{G}(\langle x\rangle)$.

Proof: Since both $F$ and $F^{\prime}$ have only one subgroup isomorphic to $T$, the isomorphism $\sigma$ fixes $T$ setwise. With our notation, $T=\langle a, b\rangle$ where $a=t_{(1,0)}$ and $b=t_{(0,1)}$. Let $a^{\sigma}=a^{u_{11}} b^{u_{12}}$ and $b^{\sigma}=a^{u_{21}} b^{u_{22}}$ for some $u_{i j} \in \mathbb{Z}_{p}$. Then $a^{\sigma}=t_{\left(u_{11}, u_{12}\right)}$ and $b^{\sigma}=t_{\left(u_{21}, u_{22}\right)}$. Moreover, it is easy to check that for any $\alpha \in V,\left(t_{\alpha}\right)^{\sigma}=t_{\alpha u}=\left(t_{\alpha}\right)^{u}$ for $u=\left(u_{i j}\right) \in G$. Because $\left(x^{-1} t_{\alpha} x\right)^{\sigma}=\left(t_{\alpha x}\right)^{\sigma}$ and $x^{\sigma} \in G$, we have $t_{\alpha u x^{\sigma}}=t_{\alpha x u}$, which implies that $x^{\sigma}=u^{-1} x u$. Therefore $\sigma=\left.I(u)\right|_{F}$. The second part of the lemma is immediate.

Lemma 3.4 Let $p$ and $q$ be two primes with $p \geq q,\left(t_{1}, t_{2}\right) \in \mathbb{Z}_{p}^{*} \times \mathbb{Z}_{q}^{*}$, and let $h=$ $\left[\left|t_{1}\right|,\left|t_{2}\right|\right]$. Define the group

$$
G\left(p, q, t_{1}, t_{2}\right)=\left\langle a, b, x \mid a^{p}=b^{q}=x^{h}=[a, b]=1, a^{x}=a^{t_{1}}, b^{x}=b^{t_{2}}\right\rangle .
$$

Then $G\left(p, q, t_{1}, t_{2}\right) \cong G\left(p, q, t_{1}^{\prime}, t_{2}^{\prime}\right)$ if and only if in $\mathbb{Z}_{p}^{*} \times \mathbb{Z}_{q}^{*}$, we have $\left\langle\left(t_{1}, t_{2}\right)\right\rangle=\left\langle\left(t_{1}^{\prime}, t_{2}^{\prime}\right)\right\rangle$ for $p \geq q$, or $\left\langle\left(t_{1}, t_{2}\right)\right\rangle=\left\langle\left(t_{2}^{\prime}, t_{1}^{\prime}\right)\right\rangle$ for $p=q$.

Proof: Let $G=G\left(p, q, t_{1}, t_{2}\right)$ and $G^{\prime}=G\left(p, q, t_{1}^{\prime}, t_{2}^{\prime}\right)$, and let us denote the three generators of $G^{\prime}$ by $a^{\prime}, b^{\prime}$ and $x^{\prime}$. We distinguish the following two cases.

Case 1. $\quad p>q$ : Assume $\left\langle\left(t_{1}, t_{2}\right)\right\rangle=\left\langle\left(t_{1}^{\prime}, t_{2}^{\prime}\right)\right\rangle$. Then $\left(t_{1}, t_{2}\right)=\left(t_{1}^{\prime}, t_{2}^{\prime}\right)^{j}$ for some $j \in \mathbb{Z}_{h}^{*}$, and the assignment $\tau: a \rightarrow a^{\prime}, b \rightarrow b^{\prime}$ and $x \rightarrow x^{\prime j}$ extends to an isomorphism $G \rightarrow G^{\prime}$. Conversely, assume that $\sigma: G \rightarrow G^{\prime}$ is an isomorphism. Then $a^{\sigma}=a^{i}, b^{\sigma}=b^{\prime j}$, 
$x^{\sigma}=a^{\prime e} b^{\prime f} x^{\prime k}$ for some $i \in \mathbb{Z}_{p}^{*}, j \in \mathbb{Z}_{q}^{*}$ and $k \in \mathbb{Z}_{h}^{*}$. Considering the action of $\sigma$ on the defining relations of $G$, one can get $t_{1} \equiv\left(t_{1}^{\prime}\right)^{k}(\bmod p)$ and $t_{2} \equiv\left(t_{2}^{\prime}\right)^{k}(\bmod q)$, which implies $\left(t_{1}, t_{2}\right) \leq\left\langle\left(t_{1}^{\prime}, t_{2}^{\prime}\right)\right\rangle$. As $\left|\left\langle\left(t_{1}, t_{2}\right)\right\rangle\right|=\left|\left\langle\left(t_{1}^{\prime}, t_{2}^{\prime}\right)\right\rangle\right|$, we get $\left\langle\left(t_{1}, t_{2}\right)\right\rangle=\left\langle\left(t_{1}^{\prime}, t_{2}^{\prime}\right)\right\rangle$.

Case 2. $p=q$ : The subcase $p=2$ is trivial. So, in what follows, we assume $p \geq 3$. The proof of sufficiency can be obtained in a similar way to Case (1), by noting the symmetry of $a$ and $b$. Conversely, assume that $\sigma: G \rightarrow G^{\prime}$ is an isomorphism. Without any loss of generality, one may assume that both $G=\langle a, b, x\rangle$ and $G^{\prime}=\left\langle a^{\prime}, b^{\prime}, x^{\prime}\right\rangle$ are subgroups of $\operatorname{AGL}(2, p)$ containing $T$. As above, we set $a=a^{\prime}=t_{(1,0)}, b=b^{\prime}=t_{(0,1)}, x=\left\|t_{1}, 0 ; 0, t_{2}\right\|$, and $x=\left\|t_{1}^{\prime}, 0 ; 0, t_{2}^{\prime}\right\|$. Then $\sigma$ fixes $T$ setwise. Since both $G$ and $G^{\prime}$ have only one conjugacy class of subgroups of order $h$, one may assume $\langle x\rangle^{\sigma}=\left\langle x^{\prime}\right\rangle$. By Lemma 3.3, $\sigma=\left.I(u)\right|_{G}$ for some inner automorphism $I(u)$ of $\operatorname{AGL}(2, p)$, where $u \in \mathrm{GL}(2, p)$ and $u^{-1} x u=x^{\prime j}$ for some integer $j$. A direct calculation shows that $u$ is of the form either $\|e, 0 ; 0 ; f\|$ or $\|0, e ; f, 0\|$. The desired result follows.

The following propositions will be used later.

Proposition 3.5 ([9, I.4.5]) Let $G$ be a group and $H \leq G$. Then $N_{G}(H) / C_{G}(H)$ is isomorphic to a subgroup of $\operatorname{Aut}(H)$.

Proposition 3.6 ([10]) Let $G$ be a finite group and $P$ a Sylow p-subgroup of $G$. If $N_{G}(P)=C_{G}(P)$, then $P$ has a normal p-complement in $G$.

Proposition 3.7 ([9, IV.2.8], [6]) Every group containing a cyclic Sylow 2-subgroup is solvable.

Proposition 3.8 ([27, 11.6, 11.7]) Every permutation group of prime degree $p$ is either insolvable and 2-transitive or isomorphic to $\mathbb{Z}_{p}: \mathbb{Z}_{s}$ for some s dividing $p-1$. Moreover, every insolvable 2-transitive group of prime degree has non-cyclic point-stabilizers.

For any odd prime $p$ and any even divisor $s$ of $p-1$, denote by $\mathcal{G}(p, s)$ the Cayley graph $\operatorname{Cay}\left(\mathbb{Z}_{p}, S\right)$, where $S$ is the subgroup of order $s$ in the group $\mathbb{Z}_{p}^{*}$.

Proposition 3.9 ([3]) $\quad$ For any odd prime $p$ and any even divisor $s$ of $p-1$, there is a unique arc-transitive graph of order $p$ and of valency $s$, up to isomorphism. It is isomorphic to the Cayley graph $\mathcal{G}(p, s)$, and its automorphism group is isomorphic to $\mathbb{Z}_{p}: \mathbb{Z}_{s}$ if $s<p-1$, and to the symmetric group $S_{p}$ if $s=p-1$.

We conclude this section by proving a group theoretical result which is crucial for the proof of the main theorem. It enables us to avoid the employment of the classification of primitive groups of degree $p$ or $p q$ for two primes $p$ and $q$. Moreover, it derives the classification theorem of regular maps in this paper independently of the classification of finite simple groups. 
Lemma 3.10 If a group $G$ contains a cyclic subgroup $H$ of index $p q$ for primes $p$ and $q$ (where $p$ may be equal to $q$ ), then $G$ is solvable.

Proof: Without any loss of generality, one may assume that $p \leq q$ and the core $H_{G}:=$ $\bigcap_{g \in G} g^{-1} \mathrm{Hg}$ is trivial, so that $G$ is isomorphic to the permutation group induced by (right) multiplication on the set $\Omega:=\{H g \mid g \in G\}$ of (right) cosets of $H$. Let $|H|=n$, so that $|G|=p q n$. If $p=q=2$, then $G$ is isomorphic to a subgroup of $S_{4}$ and so $G$ is solvable. If $p q$ or $n$ is odd, then $G$ is of odd order or $G$ contains a cyclic Sylow 2-subgroup, which implies that $G$ is solvable, by the solvability of the groups of odd order (see [6]) and Proposition 3.7. Hence, in what follows we assume that $p=2, q$ is odd and $n$ is even, so that $|\Omega|=2 q$. Our discussion is divided into the following two cases.

Case 1. $G$ acts imprimitively on $\Omega$ : Let $\mathbf{B}$ be a complete block system of $G$ and let $K$ be the kernel of $G$ on $\mathbf{B}$. If the blocks in $\mathbf{B}$ are of size $q$, then $|G / K|=2$ and $|K|=n q$. By Proposition 3.7, $K$ is solvable and consequently, $G$ is solvable. Therefore we assume that the action of $G$ induces only blocks of size 2 . Let us first deal with the case $K \neq 1$. Then $K$ is a 2-group which is transitive on each block. Set $\bar{G}=G / K$. For any block $B \in \mathbf{B}$ and any vertex $u \in B$, we have $\bar{G}_{B}=G_{B} K / K=G_{u} K / K \cong G_{u} /\left(G_{u} \cap K\right)$, a cyclic group, where $G_{u} \cong \mathbb{Z}_{n}$. Thus $\bar{G}$ is a permutation group on $\mathbf{B}$ of degree $q$, with a cyclic point-stabilizer, which by Proposition 3.8 implies that $\bar{G}$ is solvable. Consequently, $G$ is solvable as well. Next, let us consider the case $K=1$. Then $G \cong \bar{G}$ is isomorphic to a subgroup of $S_{q}$. In particular, each Sylow $q$-subgroup of $G$ has order $q$. If $\bar{G}$ is not 2-transitive on $\mathbf{B}$, then by Proposition $3.8 G \cong \bar{G}$ is solvable. Suppose that $\bar{G}$ is 2 -transitive on $\mathbf{B}$. Then $G_{B}$ is transitive on $\mathbf{B} \backslash\{B\}$, where $|\mathbf{B} \backslash\{B\}|=q-1$. Noting that $\left|G_{B}\right|=2 n$ and $\left|G_{u}\right|=n$, we have that either $G_{u}$ is transitive on $\mathbf{B} \backslash\{B\}$ or $G_{u}$ has only two orbits of cardinality $\frac{q-1}{2}$ on $\mathbf{B} \backslash\{B\}$. Since $G_{u}$ is cyclic and $K=1, G_{u}$ is intransitive on each block, which implies that $n=q-1$ or $\frac{q-1}{2}$. By Sylow's Theorem, the number of Sylow $q$-subgroups of $G$ is $q k+1$, which is a divisor of $|G|=2 n q$. Therefore, $(q k+1) \mid 2(q-1) q$ if $n=q-1$ and $(q k+1) \mid(q-1) q$ if $n=\frac{q-1}{2}$. If $k=0$, then $G$ contains only one Sylow $q$-subgroup which is normal in $G$, and so $G$ is solvable. Let $k \neq 0$. Then $(q k+1) \dagger(q-1) q$, and $(q k+1) \mid 2(q+1) q$ if and only if $k=1, q=3$ and $n=2$. In this case $G$ is a solvable group of order 12 .

Case 2. $G$ is primitive on $\Omega$ : For any group $L$, by $\pi(L)$ we denote the set of all the primes which are divisors of $|L|$. The statement will be proved by induction on $\left|\pi\left(G_{u}\right) \backslash\{2, q\}\right|$. If $\left|\pi\left(G_{u}\right) \backslash\{2, q\}\right|=0$, then $G$ is of order $2^{a} q^{b}$ for some positive integers $a$ and $b$, and consequently, $G$ is solvable (see [9, V. 7.5]). Assume $\left|\pi\left(G_{u}\right) \backslash\{2, q\}\right| \geq 1$. Take $\ell \in \pi\left(G_{u}\right) \backslash\{2, q\}$ and let $L$ be a Sylow $\ell$-subgroup of $G$ contained in $G_{u}$. Then $G_{u} \leq C_{G}(L) \leq N_{G}(L)<G$. Since $G$ is primitive, $G_{u}$ is a maximal subgroup, which implies that $G_{u}=C_{G}(L)=N_{G}(L)$, noting that $G_{u}$ is core free. By Proposition 3.6, $L$ has a normal complement $M$ in $G$. The subgroup $M$ is transitive on $\Omega$. Note that $\left|\pi\left(M_{u}\right) \backslash\{2, q\}\right|=\left|\pi\left(G_{u}\right) \backslash\{2, q\}\right|-1$, and for any $u \in \Omega, M_{u} \leq G_{u}$ is cyclic. If $M$ is primitive on $\Omega$, then, by the induction hypothesis, $M$ is solvable. If $M$ is imprimitive on $\Omega$, then by Case 1 , one can derive the solvability of $M$. Since both $M$ and $G / M \cong L$ are solvable, $G$ is solvable. 
Let us remark that there is not much room for a possible generalization of Lemma 3.10. In fact, if we replace $p q$ by $p q r$, Lemma 3.10 cannot hold. For instance, the underlying graph of the icosahedron has 12 vertices and the automorphism group of this map is $A_{5}$, the smallest nonabelian simple group.

\section{Classification theorem}

Throughout this section, let $p$ and $q$ be primes, not necessarily distinct. Recall that $\mathcal{G}(q, s)$ denotes the unique arc-transitive graph of order $q$ and valency $s$ for some even divisor $s$ of $q-1$ (see Proposition 3.9). If $s=2$, then $\mathcal{G}(q, s)$ is a cycle $C_{q}$ of order $q$. For given two graphs $\mathcal{G}$ and $\mathcal{H}$, the notation $\mathcal{G}[\mathcal{H}]$ is used to denote their lexicographic product, while $\mathcal{G} \otimes \mathcal{H}$ denotes their tensor product. In particular, $K_{n}\left[\bar{K}_{m}\right]$ is the complete $n$-partite graph $K_{m, \ldots, m}$, and $K_{2} \otimes \mathcal{G}(p, s)$ is the canonical bipartite cover of $\mathcal{G}(p, s)$, see [19] for the definition.

In order to prove the main theorem, we first introduce six different families of two generator groups $G=\langle r, \ell\rangle$ with $|\ell|=2,|r|=n,\langle r\rangle \cap\langle r\rangle^{\ell}=1$ and $|G|=n p q$. We prove in Theorem 4.1 that every arc-regular subgroup of the automorphism group of an arctransitive graph of order $p q$ with cyclic vertex-stabilizer belongs to one of these families of groups. And, for each of these groups $G$, all the corresponding algebraic maps shall be found in Lemmas 4.2-4.7. Finally, we state Theorem 4.8 establishing the classification.

(I) Let $p \geq 7, h$ any odd divisor of $p-1$ with $h \geq 3$, and let $t$ be any fixed element of order $2 h$ in $\mathbb{Z}_{p}^{*}$. Define a group

$$
G_{1}=G_{1}(p, h)=\left\langle x, y \mid x^{p}=y^{2 h}=1, x^{y}=x^{t}\right\rangle .
$$

(II) Let $p \geq 3, h$ any even divisor of $p^{2}-p$ with $h \geq 2$, and let $t$ be any fixed element of order $h$ in $\mathbb{Z}_{p^{2}}^{*}$. Define a group

$$
G_{2}=G_{2}(p, h)=\left\langle x, y \mid x^{p^{2}}=y^{h}=1, x^{y}=x^{t}\right\rangle .
$$

(III) Let $p \geq q \geq 2, p q>4$ and $\left(t_{1}, t_{2}\right) \in \mathbb{Z}_{p}^{*} \times \mathbb{Z}_{q}^{*}$ such that $t_{1} \neq t_{2}$ if $p=q$, and $\left\langle\left(t_{1}, t_{2}\right)\right\rangle$ contains $(-1,1)$ if $q=2$, and contains $(-1,-1)$ if $q \geq 3$. Let $h=\left[\left|t_{1}\right|,\left|t_{2}\right|\right]$, where $h \geq 2$ is even. Define a group

$$
G_{3}=G_{3}\left(p, q, t_{1}, t_{2}\right)=\left\langle a, b, x \mid a^{p}=b^{q}=x^{h}=[a, b]=1, a^{x}=a^{t_{1}}, b^{x}=b^{t_{2}}\right\rangle .
$$

Note that given $p$ and $q$, a necessary and sufficient condition for two such groups with different parameters $t_{1}$ and $t_{2}$ to be isomorphic has been determined in Lemma 3.4.

(IV) Let $\mathbb{F}_{p}^{*}=\langle\theta\rangle$ and let $x$ be an element of order $h$ in $\operatorname{GL}(2, p)$, where $h \geq 3$, defined as follows:

(1) If $p=2$ and $h=3$, then $x=\|1,1 ; 1,0\|$; or

(2) if $p \geq 3, h \mid\left(p^{2}-1\right)$ but $h \nmid(p-1)$, then $x=\|e, f \theta ; f, e\|$ for some fixed pair $(e, f)$ such that $|x|=h$. 
Let $T=\langle a, b\rangle$ be the translation subgroup of $\operatorname{AGL}(2, p)$ as before. Define a group

$$
G_{4}=G_{4}(p, h)=T:\langle x\rangle \leq \operatorname{AGL}(2, p) .
$$

(V) Define a group

$$
G_{5}=G_{5}(p)=\left\langle a, b, x \mid a^{p}=b^{p}=x^{2}=[a, b]=1, a^{x}=b .\right\rangle
$$

(VI) Let $\mathbb{F}_{p}^{*}=\langle\theta\rangle$ and let $H=\langle x, y\rangle$ be a subgroup in $\mathrm{GL}(2, p)$ isomorphic to a Frobenius group $\mathbb{Z}_{q}: \mathbb{Z}_{h}$ with $h \geq 2$, and two elements $x$ and $y$ are defined as follows:

(1) If $p=2, q=3$ and $h=2$, then $x=\|1,1 ; 1,0\|$ and $y=\|0,1 ; 1,0\|$;

(2) if $p>q \geq 3, q \mid(p-1)$ and $h=2$, then $x=\left\|t, 0 ; 0, t^{-1}\right\|$ where $t=\theta^{\frac{p-1}{q}}$ and $y=\|0,1 ; 1,0\|$

(3) if $p>q \geq 3, q \mid(p+1)$ and $h=2$, then $x=\|e, f \theta ; f, e\|$ for some fixed pair $(e, f)$ such that $|x|=h$, and $y=\|1,0 ;-1,0\| ;$ or

(4) if $p=q \geq 3$ and $h$ is an even divisor of $p-1$, then $x=\|1,1 ; 0,1\|$ and $y=\|1,0 ; 0, t\|$, where $t=\theta^{\frac{p-1}{h}}$.

Define a group

$$
G_{6}=G_{6}(p, q, h)=T: H \leq \operatorname{AGL}(2, p) .
$$

Theorem 4.1 Let $\mathcal{G}$ be any connected graph of order $p q$ and valency $n$, whose automorphism group $\operatorname{Aut}(\mathcal{G})$ contains an arc-regular subgroup $G$ with cyclic vertex-stabilizer. Then $G$ is isomorphic to one of the groups $G_{i}$ for $1 \leq i \leq 6$, as defined in (I)-(VI), with parameters satisfying the following conditions:

(1) $G \cong G_{1}(p, h)$, where $p \geq 7, q=2$ and $n=h \geq 3$;

(2) $G \cong G_{2}(p, h)$, where $p=q \geq 3$, and $n=h \geq 2$;

(3) $G \cong G_{3}\left(p, q, t_{1}, t_{2}\right)$, where $p \geq q \geq 2, p q>4$ and $n=h=\left[\left|t_{1}\right|,\left|t_{2}\right|\right] \geq 2$ is even;

(4) $G \cong G_{4}(p, h)$, where $p=q \geq 2, n=h \geq 3$;

(5) $G \cong G_{5}(p)$, where $p \geq 2, q=2$ and $n=p$;

(6) $G \cong G_{6}(p, q, h)$, where either $p \geq 2, q \geq 3, q \mid(p \pm 1)$ and $h=2$, or $p=q \geq 3$ and $h$ is an even divisor of $p-1$; in both cases, $n=h p$.

Moreover, for each $i$, the group $G_{i}$ is uniquely determined by its admissible parameters, and the groups $G_{i}$ are pairwise nonisomorphic.

Proof: The last statement of the theorem follows from the definitions of $G_{i}$ for $1 \leq i \leq 6$. Therefore we only need to determine the structure of the groups $G$, the arc-regular subgroups of $\operatorname{Aut}(\mathcal{G})$ with cyclic vertex-stabilizer. Clearly, $G=\langle r, \ell\rangle$, where $\ell$ is an involution and $\langle r\rangle=G_{v}$ for any fixed vertex $v$ in $V(\mathcal{G})$. By Lemma 3.10, $G$ is a solvable group of order $n p q$ with $n=\left|G_{v}\right|$. The proof is divided into two cases according to whether the action of $G$ on $V(\mathcal{G})$ is primitive or imprimitive. 
Case $1 G$ is primitive on $V(\mathcal{G})$ :

Let $N$ be a minimal normal subgroup of $G$. Then $N$ is transitive on $V(\mathcal{G})$, because $G$ is primitive. Since $G$ is solvable, $N$ is elementary abelian, which implies that $N$ acts semiregularly and so regularly on $V(\mathcal{G})$. This implies $p=q$ and $N \cong \mathbb{Z}_{p} \times \mathbb{Z}_{p}$. Therefore $G=N: G_{v}$ is isomorphic to a subgroup of order $n p^{2}$ in $\operatorname{AGL}(2, p)$, where $G_{v}$ is an irreducible cyclic subgroup of $\mathrm{GL}(2, p)$ acting on $V=\mathrm{V}(2, p)$.

Assume $p=2$, so that $G_{v} \leq \operatorname{GL}(2,2) \cong \mathbb{D}_{6}$. In this case, $G_{v}$ is the unique subgroup of order 3 of $\operatorname{GL}(2,2)$. Therefore $G \cong G_{4}(2,3)$, as defined in (4.4).

Assume $p \geq 3$. Then by Proposition $3.2(2), n \mid\left(p^{2}-1\right)$ but $n \mid(p-1)$, and GL(2,p) has only one conjugacy class of irreducible cyclic subgroups of order $n$. Therefore $\operatorname{AGL}(2, p)$ has only one conjugacy class of subgroups isomorphic to $G$ and so $G \cong G_{4}(p, h)$, as defined in (4.4) by setting $h=n \geq 3$.

Case $2 G$ is imprimitive on $V(\mathcal{G})$ :

Suppose for the moment that $G$ has a complete block system $\mathbf{B}$ with $|\mathbf{B}| \geq 3$, but does not have any complete block system consisting of only two blocks. Without any loss of generality, one may assume that $|\mathbf{B}|=q$ and $|B|=p$ for every $B \in \mathbf{B}$. Let $K$ be the kernel of $G$ acting on $\mathbf{B}$ and set $\bar{G}=G / K$. Suppose $K=1$. Then $G \cong \bar{G}$. Since $G$ is solvable, $\bar{G}$ is a solvable permutation group of prime degree $q$, which implies that $\bar{G} \cong \mathbb{Z}_{q}: \mathbb{Z}_{s}$ for a divisor $s$ of $q-1$. Now $G$ contains a normal subgroup of order $q$ which induces a block system on $V(\mathcal{G})$, say $\mathbf{B}_{1}$. Let $K_{1}$ be the kernel of $G$ on $\mathbf{B}_{\mathbf{1}}$. By the hypothesis, $G$ does not have any complete block system consisting of only two blocks, which implies $p \geq 3$. Since $q|| K_{1} \mid, K_{1}$ is transitive on each block in $\mathbf{B}_{1}$ and consequently $G / K_{1}$ acts arc-regularly on the quotient graph $\overline{\mathcal{G}}_{1}$ corresponding to $\mathbf{B}_{1}$. However $G / K_{1}$ is isomorphic to a subgroup of $\mathbb{Z}_{s}$, which implies that $G / K_{1}$ acts vertex-regularly on $\overline{\mathcal{G}}_{1}$, a contradiction. Therefore $K \neq 1$ and one can deduce that $K$ acts transitively on each block, which in turn implies that $\bar{G}$ acts arc-regularly on $\overline{\mathcal{G}}$.

From the above arguments, one can see that either $G$ has a complete block system $\mathbf{B}$ with $q=|\mathbf{B}|=2$ and the quotient graph is just an edge, or $G$ has a complete block system $\mathbf{B}$ with $q=|\mathbf{B}| \geq 3$ and $\bar{G}$ acts arc-regularly on the quotient graph $\overline{\mathcal{G}}$. From now on, we assume that $\bar{G} \cong \mathbb{Z}_{q}: \mathbb{Z}_{s}$, where if $q=2$ then $s=1$, and if $q \geq 3$ then $s$ is an even divisor of $q-1$ and the valency of $\overline{\mathcal{G}}$ is exactly $s$.

Now suppose that $K$ acts unfaithfully on a block, say $B_{0}$. Then the kernel $K_{\left(B_{0}\right)}$ of $K$ on $B_{0}$ is a nontrivial normal subgroup of $K$. Since $\overline{\mathcal{G}}$ is connected, there exist two blocks $B_{i}$ and $B_{j}$ such that $\left\{B_{i}, B_{j}\right\} \in E(\overline{\mathcal{G}})$ and $K_{\left(B_{0}\right)}$ fixes $B_{i}$ pointwise and is transitive on $B_{j}$. It implies that the induced subgraph $\mathcal{G}\left(B_{i} \cup B_{j}\right) \cong K_{p, p}$. Therefore $n=s p$ and $K \cong \mathbb{Z}_{p} \times \mathbb{Z}_{p}$. Since $\overline{\mathcal{G}}$ is arc-transitive, it follows that $\mathcal{G} \cong \mathcal{G}(q, s)\left[\bar{K}_{p}\right]$ is the lexicographic product of the (unique) connected arc-transitive graph $\mathcal{G}(q, s)$ of order $q$ and valency $s$ with the complement of the complete graph of order $p$. In this case, it has been proved in [5] that $G$ is isomorphic to $G_{2}(p, h)$ for $p \mid h$, as in (4.2) where $h=n$, or to $G_{5}(p)$ as in (4.5) where $n=p$, or to $G_{6}(p, q, h)$ as in (4.6) where $n=p h$.

Next, suppose that $K$ acts faithfully on each block. Then we have $K_{v} \cong \mathbb{Z}_{\frac{n}{s}}$ and $K \cong$ $\mathbb{Z}_{p}: \mathbb{Z}_{\frac{n}{s}}$, because $G_{v}$ is cyclic and $K$ is faithful. In what follows, we assume that ${ }^{s} P=\langle x\rangle$ is 
the Sylow $p$-subgroup of $K$, where $|P|=p$. Note that $P$ is also normal in $G$, because it is a characteristic subgroup of $K$. If $p=q=2$, then $G$ is primitive on $V(\Gamma)$. Therefore we assume that $p q \neq 4$ and distinguish the following three cases: $(i) p>q=2$; (ii) $q \geq 3$ and $p \neq q$; and (iii) $q=p \geq 3$.

Subcase (i) $p>q=2$ : In this case, $G$ is an extension of $K \cong \mathbb{Z}_{p}: \mathbb{Z}_{n}$ by $\mathbb{Z}_{2}$, and $G_{v} \cong \mathbb{Z}_{n}$, where $n \mid(p-1)$. Since $(p, 2 n)=1$ and $G$ is solvable, $P$ has a complement, say $H$, in $G$.

If $C_{G}(P)=P$, then by Proposition $3.5, H \cong G / P=G / C_{G}(P)$ is isomorphic to a subgroup of $\operatorname{Aut}(P) \cong \mathbb{Z}_{p-1}$, which implies $H \cong \mathbb{Z}_{2 n}$. Thus $G \cong \mathbb{Z}_{p}: \mathbb{Z}_{2 n}$, a Frobenius group. Assume that $G=\langle x\rangle:\langle y\rangle$, where $|x|=p$ and $|y|=2 n$. Since $G$ has only one conjugacy class of cyclic subgroups of order $2 n$, one may assume that $r=y^{2 i}$ and $\ell=x^{j} y^{n}$, where $i \in \mathbb{Z}_{n}^{*}$ and $j \in \mathbb{Z}_{p}^{*}$, so that $G=\langle r, \ell\rangle$ and $\ell^{2}=1$. If $n$ is even, then $\langle r, \ell\rangle \leq\left\langle x, y^{2}\right\rangle \neq G$. Hence $n$ must be odd. Therefore $G \cong G_{1}(p, h)$, as defined in (4.1), by setting $h=n \geq 3$.

If $C_{G}(P) \neq P$, then $\left|C_{G}(P)\right|=2 p$ as $C_{K}(P)=P$. Moreover, $C_{G}(P)=P \times\langle b\rangle$ for some involution $b$, which implies that $\langle b\rangle$ is normal in $G$, and so it is contained in the center of $G$. Setting $h=n \geq 2$, we get $G \cong G_{3}\left(p, 2, t_{1}, 1\right)$ as defined in (4.3). Since $\ell \in G \backslash\langle b\rangle$, it follows that $n$ must be even.

Subcase (ii) $q \geq 3$ and $p \neq q$ : Since $K$ is assumed to be faithful, we have $K \cap C_{G}(P)=P$, where $|P|=p$ as mentioned before. Suppose that $C_{G}(P) \leq K$. Then $C_{G}(P)=P$. By using Proposition 3.5 one can then see that $G / P=G / C_{G}(P)$ is isomorphic to a subgroup of $\operatorname{Aut}(P) \cong \mathbb{Z}_{p-1}$, which is cyclic. However, from $(G / P) /(K / P) \cong G / K \cong \mathbb{Z}_{q}: \mathbb{Z}_{s}$ where $s \geq 2$, one can deduce that $G / P$ cannot be cyclic, a contradiction. Therefore $C_{G}(P) \supsetneqq K$, which implies that $C_{G}(P)$ acts transitively on $V(\mathcal{G})$. Let $M=O_{p, q}\left(C_{G}(P)\right)$ ). (Here, with the notation $O_{p}(G)$ for the maximal normal $p$-subgroup of $G, O_{p, q}\left(C_{G}(P)\right)$ denotes the subgroup of $C_{G}(P)$ such that $O_{p, q}\left(C_{G}(P)\right) / O_{p}\left(C_{G}(P)\right)=O_{q}\left(C_{G}(P) / O_{p}\left(C_{G}(P)\right)\right)$ ). Then $M \cong \mathbb{Z}_{p} \times \mathbb{Z}_{q}$ is a normal subgroup of $G$, which acts regularly on $V$. Let $Q$ be a subgroup of $M$ of order $q$. Then $M=P \times Q$. Therefore $G=(P \times Q): G_{v} \leq\left(\mathbb{Z}_{p} \times \mathbb{Z}_{q}\right): \mathbb{Z}_{n}$, where $\mathbb{Z}_{n}$ is isomorphic to a subgroup of $\operatorname{Aut}\left(\mathbb{Z}_{p} \times \mathbb{Z}_{q}\right) \cong \mathbb{Z}_{p}^{*} \times \mathbb{Z}_{q}^{*}$. Taking into account the symmetry of $P$ and $Q$ in this case, one may assume $p>q \geq 3$, noting that if $p=2$ then we are back to the second case in Subcase $(i)$. Now, let $P=\langle a\rangle, Q=\langle b\rangle$ and $G_{v}=\langle x\rangle$, and suppose that $a^{x}=a^{t_{1}}$ and $b^{x}=b^{t_{2}}$, where $\left(t_{1}, t_{2}\right) \in \mathbb{Z}_{p}^{*} \times \mathbb{Z}_{q}^{*}$. Then $n=|x|=\left[\left|t_{1}\right|,\left|t_{2}\right|\right]$. Suppose $G=\langle r, \ell\rangle$ for an involution $\ell$, where $r \in\langle x\rangle$. Then $\ell=a^{i} b^{j} x^{\frac{n}{2}}$ for some $i \in \mathbb{Z}_{p}^{*}$ and $j \in \mathbb{Z}_{q}^{*}$. From $\ell^{2}=1$, we have $t_{1}^{\frac{n}{2}}=-1$ in $\mathbb{Z}_{p}$ and $t_{2}^{\frac{n}{2}}=-1$ in $\mathbb{Z}_{q}$, which means $(-1,-1) \leq\left\langle\left(t_{1}, t_{2}\right)\right\rangle$. Therefore for $i=1$ and $2,\left|t_{i}\right|$ is even and $\left|t_{i}\right| /\left(\left|t_{1}\right|,\left|t_{2}\right|\right)$ is odd. Thus setting $h=n \geq 2$, one can have $G \cong G_{3}\left(p, q, t_{1}, t_{2}\right)$ for $p>q \geq 3$, as defined in (4.3).

Subcase (iii) $q=p \geq 3$ : In this case, the Sylow $p$-subgroup $P$ of $G$ is a normal subgroup of $G$ and its order is $p^{2}$. Hence $G=P: G_{v}$.

If $P \cong \mathbb{Z}_{p^{2}}$, then $G \cong \mathbb{Z}_{p^{2}}: \mathbb{Z}_{n} \leq \mathbb{Z}_{p^{2}}: \operatorname{Aut}\left(\mathbb{Z}_{p^{2}}\right)$, where $n \mid(p-1)$ as $p \nmid n$. Since $\operatorname{Aut}\left(\mathbb{Z}_{p^{2}}\right) \cong \mathbb{Z}_{p^{2}-p}$, a cyclic group, and all the complements of $P$ in $G$ are conjugate, we deduce that $G \cong G_{2}(p, h)$ for $h \mid(p-1)$ as defined in (4.2), by setting $h=n \geq 2$. 
If $P \cong \mathbb{Z}_{p}^{2}$, then $G=P: G_{v} \cong \mathbb{Z}_{p}^{2}: \mathbb{Z}_{n} \leq \operatorname{AGL}(2, p)$, and by Lemma 3.2(3) $G_{v}=\langle x\rangle$ can be chosen to be a diagonal subgroup not contained in $Z$. Let $x=\left\|t_{1}, 0 ; 0, t_{2}\right\|$, and let $\ell=a^{i} b^{j} x^{\frac{n}{2}}$ be an involution for some $i, j \in \mathbb{Z}_{p}^{*}$. Then from $\ell^{2}=1$, we have $x^{\frac{n}{2}}=z$, the central involution of GL(2,p), which implies that $\left|t_{i}\right| /\left(\left|t_{1}\right|,\left|t_{2}\right|\right)$ is odd for $i=1,2$. Therefore $G \cong G_{3}\left(p, p, t_{1}, t_{2}\right)$, by setting $h=n>2$, as defined in (4.3).

Our next task is to determine all the nonisomorphic algebraic maps coming from the groups $G=G_{i}$ for $1 \leq i \leq 6$. By the results of Section 2, we only need to determine all the representatives of the orbits of the action of $\operatorname{Aut}(G)$ on the set of the generating pairs $(r, \ell)$ of $G$ satisfying $|\ell|=2,|r|=n,|G|=n p q$ and $\langle r\rangle \cap\langle r\rangle^{\ell}=1$. This will be carried out case by case in Lemmas 4.2-4.7. Recall that $\phi(h)$ denotes the Euler function, that is, the number of positive integers less than $h$ which are coprime to $h$.

Lemma 4.2 Let $G_{1}=G_{1}(p, h)$ be a group as defined in (4.1). Then any regular map $\mathcal{M}\left(G_{1} ; r, \ell\right)$ with underlying graph $\mathcal{G}$ of order $2 p$ is isomorphic to one of the following $\phi(h)$ regular maps:

$$
\mathcal{M}_{1}=\mathcal{M}_{1}(p, h, i)=\mathcal{M}\left(G_{1} ; y^{2 i}, x y^{h}\right),
$$

where $p \geq 7, h \geq 3, h$ is an odd divisor of $p-1$, and $i \in \mathbb{Z}_{h}^{*}$. Moreover, $\mathcal{G}$ is a bipartite graph of valency $h$.

Proof: Recall that

$$
G_{1}=G_{1}(p, h)=\left\langle x, y \mid x^{p}=y^{2 h}=1, x^{y}=x^{t}\right\rangle .
$$

In this case, $q=2$ and $n=|r|=h \geq 3$. In what follows, we find all the representatives of the orbits of $\operatorname{Aut}\left(G_{1}\right)$ on the set of admissible generating pairs $(r, \ell)$ of $G_{1}$. Since $G_{1}$ has only one conjugacy class of involutions and one conjugacy class of subgroups of order $h$, one may assume $r=y^{2 i}$ for any $i \in \mathbb{Z}_{h}^{*}$. Moreover, each involution in $G_{1} \backslash\langle y\rangle$ has the form $x^{j} y^{h}$ for $j \in \mathbb{Z}_{p}^{*}$ and the automorphism $\tau$ of $G_{1}$ defined by $x^{\tau}=x^{j}$ and $y^{\tau}=y$ fixes $r$ and sends $x y^{h}$ to $x^{j} y^{h}$. Therefore we may fix $\ell=x y^{h}$.

Now, suppose that $\sigma \in \operatorname{Aut}\left(G_{1}\right)$ fixes $\left\langle y^{2}\right\rangle$ setwise and $\langle\ell\rangle$ pointwise. Then $x^{\sigma}=x$ and $y^{\sigma}=y^{j}$ for some $j \in \mathbb{Z}_{2 h}^{*}$. Since $\sigma$ preserves the relation $x^{y}=x^{t}$, we have $y^{-j} x y^{j}=x^{t}$ and so $t^{j} \equiv t(\bmod p)$, which implies that $j=1$ in $\mathbb{Z}_{2 h}^{*}$ and consequently, $\sigma=1$. Therefore we have $\phi(h)$ choices for $(r, \ell)$ and so obtain $\phi(h)$ nonisomorphic regular maps as claimed in (4.7).

Lemma 4.3 Let $G_{2}=G_{2}(p, h)$ be a group as defined in (4.2). Then any regular map $\mathcal{M}\left(G_{2} ; r, \ell\right)$ with underlying graph $\mathcal{G}$ of order $p^{2}$ is isomorphic to one of the following $\phi(h)$ regular maps:

$$
\mathcal{M}_{2}=\mathcal{M}_{2}(p, h, i)=\mathcal{M}\left(G_{2} ; y^{i}, x y^{\frac{h}{2}}\right),
$$

where $p \geq 3, h \geq 2, h$ is an even divisor of $p^{2}-p$, and $i \in \mathbb{Z}_{h}^{*}$. Moreover, if $p \mid h$ then $\mathcal{G} \cong \mathcal{G}\left(p, \frac{h}{p}\right)\left[\bar{K}_{p}\right] ;$ and if $(p, h)=1$ then $h \mid(p-1)$ and $\mathcal{G}$ is a $p$-fold cover of the graph $\mathcal{G}(p, h)$. 
Proof: Recall that

$$
G_{2}=G_{2}(p, h)=\left\langle x, y \mid x^{p^{2}}=y^{h}=1, x^{y}=x^{t}\right\rangle
$$

In this case, $p=q$ and $n=h$. Applying a similar argument to Lemma 4.2, one can show that all the admissible pairs $(r, \ell)$ are of the form $r=y^{i}$ and $\ell=x y^{\frac{h}{2}}$ for $i \in \mathbb{Z}_{h}^{*}$. Hence there are exactly $\phi(h)$ nonisomorphic regular maps, as shown in (4.8). Moreover, the structure of the underlying graph $\mathcal{G}$ can be seen from the coset graph.

Lemma 4.4 Let $G_{3}=G_{3}\left(p, q, t_{1}, t_{2}\right)$ be a group as defined in (4.3). Then any regular map $\mathcal{M}\left(G_{3} ; r, \ell\right)$ with underlying graph $\mathcal{G}$ of order pq is isomorphic to one of the following regular maps:

$$
\mathcal{M}_{3}=\mathcal{M}_{3}\left(p, q, t_{1}, t_{2}, i\right)=\mathcal{M}\left(G_{3} ; x^{i}, a b x^{\frac{h}{2}}\right),
$$

where $p \geq q \geq 2, p q>4$ and $\left(t_{1}, t_{2}\right) \in \mathbb{Z}_{p} \times \mathbb{Z}_{q}$ such that $t_{1} \neq t_{2}$ if $p=q$, and $\left\langle\left(t_{1}, t_{2}\right)\right\rangle$ contains $(-1,1)$ if $q=2$, and contains $(-1,-1)$ if $q \geq 3$; and $i \in \mathbb{Z}_{h}^{*} /\left(\mathbb{Z}_{h}^{*}\right)^{2+}$ if $p=q$ and $h=\left|t_{1}\right|=\left|t_{2}\right|$, and $i \in \mathbb{Z}_{h}^{*}$ otherwise.

Hence we have $\phi(h) / \mid\left(\mathbb{Z}_{h}^{*}\right)^{2+}$ or $\phi(h)$ nonisomorphic regular maps according to whether $p=q$ and $\left|t_{1}\right|=\left|t_{2}\right|$ or not.

Proof: Recall that

$$
G_{3}=G_{3}\left(p, q, t_{1}, t_{2}\right)=\left\langle a, b, x \mid a^{p}=b^{q}=x^{h}=[a, b]=1, a^{x}=a^{t_{1}}, b^{x}=b^{t_{2}}\right\rangle .
$$

The proof is divided into three cases to be discussed separately.

Case $1 p>q=2$ : Now $\left|t_{2}\right|=1$ and $h=\left|t_{1}\right|$. In this case, $b \in Z\left(G_{3}\right)$, so $G_{3}$ has only one class of cyclic subgroups of order $h$. Hence one may assume $r=x^{i}$ for $i \in \mathbb{Z}_{h}^{*}$ and $\ell=a^{j} b x^{\frac{h}{2}}$ for $j \in \mathbb{Z}_{p}^{*}$. Since the automorphism $\tau$ of $G$ defined by $a^{\tau}=a^{j}, b^{\tau}=b$ and $x^{\tau}=x$ fixes $r$ and sends $a b x^{\frac{h}{2}}$ to $a^{j} b x^{\frac{h}{2}}$, one may fix $\ell=a b x^{\frac{h}{2}}$. Similarly, one can show that if some $\sigma$ in $\operatorname{Aut}\left(G_{3}\right)$ fixes $\langle\ell\rangle$ pointwise and $\langle r\rangle$ setwise, then it must be the identity. Therefore we obtain $\phi(h)$ nonisomorphic regular maps $\mathcal{M}\left(p, 2, t_{1}, 1, i\right)$ as claimed in (4.9).

Case $2 p>q \geq 3$ : Now both $\left|t_{1}\right|$ and $\left|t_{2}\right|$ are even and $\left|t_{i}\right|^{\frac{h}{2}}=-1$. Let $P=\langle a\rangle$ and $Q=$ $\langle b\rangle$. We consider $G_{3}$ as a subgroup of $A:=(P \times Q)$ : Aut $(P \times Q) \cong\left(\mathbb{Z}_{p} \times \mathbb{Z}_{q}\right):\left(\mathbb{Z}_{p}^{*} \times \mathbb{Z}_{q}^{*}\right)$.

Note that each cyclic subgroup $H$ of order $h$ is a complement of $P \times Q$ in $G_{3}$ and $H$ induces a faithful automorphism action on it. Hence one may assume that $r \in\langle x\rangle$. Thus $\ell$ has to be of the form $a^{i} b^{j} x^{\frac{h}{2}}$ for some $i \in \mathbb{Z}_{p}^{*}$ and $j \in \mathbb{Z}_{q}^{*}$. Since the action of the subgroup of inner automorphisms $I(g)$ for $g \in \operatorname{Aut}(P \times Q)$ of $A$ fixes $x$ and maps $a b$ to $a^{i} b^{j}$, one may fix $\ell=a b x^{\frac{h}{2}}$. Suppose $\sigma \in \operatorname{Aut}\left(G_{3}\right)$ fixes $\langle\ell\rangle$ pointwise and $\langle x\rangle$ setwise. Then $\sigma$ satisfies: $a^{\sigma}=a, b^{\sigma}=b$ and $x^{\sigma}=x^{k}$ for some $k \in \mathbb{Z}_{h}^{*}$. Since $\sigma$ preserves the defining relations $a^{x}=a^{t_{1}}$ and $b^{x}=b^{t_{2}}$, one can get $t_{1}^{k-1} \equiv 1(\bmod p)$ and $t_{2}^{k-1} \equiv 1(\bmod q)$, which 
implies that $\left|t_{1}\right| \mid(k-1)$ and $\left|t_{2}\right| \mid(k-1)$ and so $h \mid(k-1)$, implying $k=1$ in $\mathbb{Z}_{h}^{*}$, or equivalently, $\sigma=1$. Hence we obtain $\phi(h)$ nonisomorphic regular maps as required.

Case $3 p=q \geq 3$ : Let $G=\mathrm{GL}(2, p)$ and let $D$ be the diagonal subgroup of $G$. In this case, by identifying $a$ with $t_{(1,0)}, b$ with $t_{(0,1)}$, and $x$ with $\left\|t_{1}, 0 ; 0, t_{2}\right\| \in D \backslash Z(G)$, one may consider $G_{3}$ as a subgroup of $A:=\operatorname{AGL}(2, p)$ satisfying $G_{3}=T:\langle x\rangle$. Set $z=x^{\frac{h}{2}}$, which is the central involution of $G$. Since $G_{3}$ has only one conjugacy class of subgroups of order $h$ and of order 2, respectively, one may assume that $r \in\langle x\rangle$. Therefore $\ell$ is of the form $a^{i} b^{j} z$ for some $i, j \in \mathbb{Z}_{p}$, because $x$ fixes $(1,0)$ and $(0,1)$. Since the action of the subgroup of inner automorphisms $I(g)$ of $A$ for $g \in D$ fixes $r$ and is transitive on the set $\left\{t_{(i, j)} \mid i, j \in \mathbb{Z}_{p}^{*}\right\}$, one may fix $\ell=a b z$. Suppose that $1 \neq \sigma \in \operatorname{Aut}\left(G_{3}\right)$ fixes $\langle x\rangle$ setwise and $\langle\ell\rangle$ pointwise. Then $\sigma$ fixes $a b$. By Lemma 3.3, we have $\sigma=\left.I(u)\right|_{G_{3}} \in \operatorname{Inn}(A)$ for some $u \in N_{G}(\langle x\rangle)$. By Proposition 3.2(2), $C_{G}(\langle x\rangle)=D$ and $N_{G}(\langle x\rangle)=D:\langle y\rangle$, where $y=\|0,1 ; 1,0\|$. It follows that either $u=\|r, 0 ; 0 ; s\|$ or $u=\|0, r ; s, 0\|$ for some $r, s$ in $\mathbb{Z}_{p}^{*}$. Because $t_{(1,1) u}=(a b)^{u}=(a b)^{\sigma}=a b=t_{(1,1)}$, we have $r=s=1$ and so $u=y$, noting that $\sigma \neq 1$. Therefore $x^{\sigma}=x^{y}=\left\|t_{2}, 0 ; t_{1}, 0\right\|$, which implies that $\left|t_{1}\right|=\left|t_{2}\right|$ and so $h=\left|t_{i}\right|$. Assume $x^{\sigma}=x^{j}$. Then $t_{2}=t_{1}^{j}=t_{2}^{j^{2}}$ and so $j^{2}=1$ in $\mathbb{Z}_{h}^{*}$. Therefore in the case $p=q$ and $h=\left|t_{i}\right|$, one may assume that $r=x^{i}$ for some $i \in \mathbb{Z}_{h}^{*} /\left(\mathbb{Z}_{h}^{*}\right)^{2+}$. In the other cases, $r=x^{i}$ for some $i \in \mathbb{Z}_{h}^{*}$.

Lemma 4.5 Let $G_{4}=G_{4}(p, n)$ be a group as defined in (4.4). Then any regular map $\mathcal{M}\left(G_{4} ; r, \ell\right)$ with underlying graph $\mathcal{G}$ of order $p^{2}$ is isomorphic to one of the following $\phi(h) /\left|\left(\mathbb{Z}_{h}^{*}\right)^{2+}\right|$ nonisomorphic regular maps

$$
\mathcal{M}_{4}=\mathcal{M}_{4}(p, h, i)=\mathcal{M}\left(G_{4}, x^{i}, a z\right)
$$

where $h \mid\left(p^{2}-1\right)$ but $h \nmid(p-1)$ and $h \geq 3$; either $z=1$ for $p=2$ or $z=x^{\frac{h}{2}}$ for $p \geq 3$; and $i \in \mathbb{Z}_{h}^{*} /\left(\mathbb{Z}_{h}^{*}\right)^{2+}$.

Proof: If $p=2$ and $h=3$, then $G_{4} \cong A_{4}$. In this case, we have only one regular map $\mathcal{M}_{4}(2,3,1)$ as expected.

Assume that $p \geq 3$. In a similar way to the proof of Case 3 of Lemma 4.4, we let $G=$ $\mathrm{GL}(2, p)$, and identifying respectively $a$ with $t_{(1,0)}, b$ with $t_{(0,1)}$, and $x$ with $\|e, f \theta ; f, e\|$ for some fixed pair $(e, f)$ such that $e f \neq 0$ and $x$ is of order $h$, we consider $G_{4}$ as a subgroup of $A:=\operatorname{AGL}(2, p)$ of the form $G_{4}=T:\langle x\rangle$. Then the central involution $z=x^{\frac{h}{2}} \neq 1$. Since $G_{4}$ has only one conjugacy class of subgroups of order $h$ and one of order 2, one may assume that $r \in\langle x\rangle$. As well, one may assume that $\ell=a^{i} b^{j} z$ for $i, j \in \mathbb{Z}_{p}$ with $(i, j) \neq(0,0)$. By Lemma 3.2(2), all the elements of the form $\left\|e^{\prime}, f^{\prime} \theta ; f^{\prime}, e^{\prime}\right\|$ form a cyclic subgroup $H$ of $G$, which is regular on $V \backslash\{0\}$. Therefore the subgroup of $A$ consisting of the inner automorphisms $I(g)$ for $g \in H$ is transitive on the set $\left\{a^{i} b^{j} z \mid i, j \in \mathbb{Z}_{p},(i, j) \neq(0,0)\right\}$. Therefore one may fix $\ell=a z$.

Suppose that $1 \neq \sigma \in \operatorname{Aut}\left(G_{4}\right)$ fixes $\langle x\rangle$ setwise and $\langle\ell\rangle$ pointwise. Then $\sigma$ fixes $a$. By Lemma 3.3, $\sigma=\left.I(u)\right|_{G_{4}} \in \operatorname{Inn}\left(G_{4}\right)$ for some $u \in N_{G}(\langle x\rangle)$. By Proposition 3.2(2), $C_{G}(\langle x\rangle)=H$ and $N_{G}(\langle x\rangle)=H:\langle y\rangle$, where $y=\|1,0 ; 0,-1\|$. Hence $u=\|e, f \theta ; f, e\|$ 
or $\|e,-f \theta ; f,-e\|$ for the above fixed pair $(e, f)$. Computing $a^{\sigma}=a$, we get $f=0$ and thus $u=y$. Therefore $x^{\sigma}=x^{y}=\|e,-f \theta ;-f, e\|$. In particular, $|\sigma|=2$. Assume $x^{\sigma}=x^{j}$. Then $j^{2}=1$ in $\mathbb{Z}_{h}^{*}$. Consequently, one may assume that $r=x^{i}$ for $i \in \mathbb{Z}_{h}^{*} /\left(\mathbb{Z}_{h}^{*}\right)^{2+}$. Hence we obtain $\phi(h) /\left|\left(\mathbb{Z}_{h}^{*}\right)^{2+}\right|$ nonisomorphic regular maps as required for (4.10).

The following two lemmas dealing with the families of groups $G_{5}(p)$ and $G_{6}(p, q, h)$ are proved in [5].

Lemma 4.6 Let $G_{5}(p)$ be a group as defined in (4.5), where $p \geq 2$. Then any regular map $\mathcal{M}\left(G_{5} ; r, \ell\right)$ with underlying graph of order $2 p$ is isomorphic to the map

$$
\mathcal{M}_{5}(p)=\mathcal{M}\left(G_{5} ; a, x\right),
$$

and its underlying graph is $K_{p, p}$.

Lemma 4.7 Let $G_{6}=G_{6}(p, q, h)$ be a group as defined in (4.6). Then any regular map $\mathcal{M}\left(G_{6} ; r, \ell\right)$ with underlying graph $\mathcal{G}$ of order $p q$ is isomorphic to one of the following $\frac{q-1}{h} \phi(h)$ nonisomorphic regular maps

$$
\mathcal{M}_{6}(p, q, h, i, j):=\mathcal{M}\left(G_{6} ; a^{\prime} y^{j}, x^{i} y^{\frac{h}{2}}\right),
$$

where either $p \geq 2, q \geq 3, q \mid(p \pm 1)$ and $h=2$, or $p=q \geq 3$ and $h$ is an even divisor of $q-1$. In both cases, $i \in \mathbb{Z}_{q}^{*} /\left(\mathbb{Z}_{q}^{*}\right)^{h+}$ and $j \in \mathbb{Z}_{h}^{*}$; and

$$
a^{\prime}= \begin{cases}t_{(1,0)} & \text { if } p=2, q \mid(p+1) \text { or } p=q, \\ t_{(1,1)} & \text { if } q \mid(p-1) .\end{cases}
$$

Moreover, $\mathcal{G} \cong \mathcal{G}(q, h)\left[\bar{K}_{p}\right]$.

By combining Theorem 4.1 and Lemmas 4.2-4.7, the following classification theorem is obtained.

Theorem 4.8 Let $\mathcal{M}$ be a regular map with a simple underlying graph $\mathcal{G}$ of order $p q$ for any two primes $p$ and $q$ with $p \geq q$. Then $\mathcal{M}$ is isomorphic to one of the following regular maps uniquely determined by the given integer parameters:

(1) $p=q=2$ :

$\mathcal{M} \cong \mathcal{M}_{5}(2)$ and $\mathcal{G} \cong C_{4}$.

$\mathcal{M} \cong \mathcal{M}_{4}(2,3,1)$ and $\mathcal{G} \cong K_{4}$.

(2) $p=q \geq 3$ :

$\mathcal{M} \cong \mathcal{M}_{2}(p, h, i)$, where $h \geq 2, h$ is an even divisor of $p(p-1)$ and $i \in \mathbb{Z}_{h}^{*}$. The graph $\mathcal{G}$ is isomorphic to $\mathcal{G}(p, h / p)\left[\bar{K}_{p}\right]$ if $p \mid h$, or to a p-fold regular cover of $\mathcal{G}(p, h)$ if $p \nmid h$.

$\mathcal{M} \cong \mathcal{M}_{3}\left(p, p, t_{1}, t_{2}, i\right)$, where $t_{1} \neq t_{2},\left\langle\left(t_{1}, t_{2}\right)\right\rangle$ is a subgroup in $\mathbb{Z}_{p}^{*} \times \mathbb{Z}_{p}^{*}$ containing $(-1,-1)$ and $h=\left[\left|t_{1}\right|,\left|t_{2}\right|\right]>2 ;$ and $i \in \mathbb{Z}_{h}^{*} /\left(\mathbb{Z}_{h}^{*}\right)^{2+}$ if $h=\left|t_{1}\right|=\left|t_{2}\right|$, and $i \in \mathbb{Z}_{h}^{*}$ otherwise. The graph $\mathcal{G}$ is of valency $h$ and $\operatorname{Aut}(\mathcal{M})$ acts imprimitively on $V(\mathcal{G})$. 
$\mathcal{M} \cong \mathcal{M}_{4}(p, h, i)$, where $h \geq 3, h \mid\left(p^{2}-1\right)$ but $h \nmid(p-1), i \in \mathbb{Z}_{h}^{*} /\left(\mathbb{Z}_{h}^{*}\right)^{+2}$. The graph $\mathcal{G}$ is of valency $h$ and $\operatorname{Aut}(\mathcal{M})$ acts primitively on $V(\mathcal{G})$.

$\mathcal{M} \cong \mathcal{M}_{6}(p, p, h, i, j)$, where $h$ is an even divisor of $p-1, i \in \mathbb{Z}_{p}^{*} /\left(\mathbb{Z}_{p}^{*}\right)^{h+}$, and $j \in \mathbb{Z}_{h}^{*}$. Moreover, $\mathcal{G} \cong \mathcal{G}(p, h)\left[\bar{K}_{p}\right]$.

(3) $p \geq 3$ and $q=2$ :

$\mathcal{M} \cong \mathcal{M}_{6}(2,3,2,1,1)$, where $p=3 . \mathcal{G} \cong \mathcal{G}(3,2)\left[\bar{K}_{2}\right]$.

$\mathcal{M} \cong \mathcal{M}_{1}(p, h, i)$, where $p \geq 7, h \geq 3, h$ is an odd divisor of $p-1$ and $i \in \mathbb{Z}_{h}^{*}$. The graph $\mathcal{G}$ is bipartite of valency $h$.

$\mathcal{M} \cong \mathcal{M}_{3}\left(p, 2, t_{1}, 1, i\right)$, where $p \geq 3, t_{1} \in \mathbb{Z}_{p}^{*}, i \in \mathbb{Z}_{h}^{*}$ for $h=\left|t_{1}\right|$, an even divisor of $p-1 . \mathcal{G} \cong K_{2} \otimes \mathcal{G}(p, h)$ is a bipartite graph of valency $h$.

$\mathcal{M} \cong \mathcal{M}_{5}(p)$, where $p \geq 3$ and $\mathcal{G} \cong K_{p, p}$.

(4) $p>q \geq 3$ :

$\mathcal{M} \cong \mathcal{M}_{3}\left(p, q, t_{1}, t_{2}, i\right)$, where $\left\langle\left(t_{1}, t_{2}\right)\right\rangle$ is a subgroup in $\mathbb{Z}_{p}^{*} \times \mathbb{Z}_{q}^{*}$ containing $(-1,-1)$ and $i \in \mathbb{Z}_{h}^{*}$ for $h=\left[\left|t_{1}\right|,\left|t_{2}\right|\right]$. The graph $\mathcal{G}$ is of valency of $h$.

$\mathcal{M}_{6}(p, q, 2, i, 1)$, where $q \mid(p \pm 1)$ and $i \in \mathbb{Z}_{q}^{*} /\left(\mathbb{Z}_{q}^{*}\right)^{2+}$. Moreover, $\mathcal{G} \cong C_{q}\left[\bar{K}_{p}\right]$.

It follows that in the (general) case (4) of the theorem, where $p>q \geq 3$, only two sorts of regular maps are admissible. One is formed by regular embeddings of the lexicographic products of cycles $C_{q}$ with $p$ isolated vertices. The maps in the other family, denoted as $\mathcal{M}_{3}\left(p, q, t_{1}, t_{2}, i\right)$, are the least regular maps covering both a regular embedding of $\mathcal{G}\left(p, h /\left|t_{2}\right|\right)$ and one of $\mathcal{G}\left(q, h /\left|t_{1}\right|\right)$.

For $p \geq q \geq 3$, let $S_{h}(p, q)$ be the set of the cyclic subgroups $H$ of order $h$ of $\mathbb{Z}_{p}^{*} \times \mathbb{Z}_{q}^{*}$ that contain $(-1,-1)$, and let $S_{h}^{\prime}(p)$ be the set of the cyclic subgroups $H=\left\langle\left(t, t^{j}\right)\right\rangle$ of $\mathbb{Z}_{p}^{*} \times \mathbb{Z}_{p}^{*}$ for $j \in \mathbb{Z}_{h}^{*} /\left(\mathbb{Z}_{h}^{*}\right)^{2+}$, where $h=|t|$. Then $S_{h}(p, q)$ and $S_{h}^{\prime}(p)$ can be easily determined for any given $p$ and $q$ and $h$. Finally, define $S(p, q)=\cup_{h} S_{h}(p, q)$ and $S^{\prime}(p)=\cup_{h} S_{h}^{\prime}(p)$. Using this notation we obtain the following enumeration of nonisomorphic regular embeddings with underlying graphs of order $p q$.

Corollary 4.9 Let $p$ and $q$ be any two primes with $p \geq q$. Let $N(p, q)$ be the number of all regular embeddings with underlying graphs of order $p q$. Then

(1) $N(2,2)=2, N(3,2)=3$, and $N(p, 2)=1+\sum_{h \mid(p-1), h \geq 2} \phi(h)$, if $p \geq 5$;

(2) $N(p, p)=\sum_{h|(p-1), 2| h} \phi(h)\left(p+\frac{p-1}{h}\right)+\sum_{h\left|\left(p^{2}-1\right), h \nmid(p-1), 2\right| h} \phi(h) /\left|\left(Z_{h}^{*}\right)^{2+}\right|$ $+\sum_{H \in S(p, p) \backslash S^{\prime}(p)} \phi(|H|)+\sum_{H \in S^{\prime}(p)} \phi(|H|) /\left|\left(Z_{|H|}^{*}\right)^{2+}\right|$, if $p \geq 3$;

(3) $N(p, q)=\sum_{H \in S(p, q)} \phi(|H|)$, if $p>q \geq 3, q \nmid(p+1)$ and $q \nmid(p-1)$;

(4) $N(p, q)=\frac{q-1}{2}+\sum_{H \in S(p, q)} \phi(|H|)$, if $p>q \geq 3$, and either $q \mid(p-1)$ or $q \mid(p+1)$. 


\section{Genera of regular maps with $p q$ vertices}

An orientable map $\mathcal{M}=\mathcal{M}(\mathcal{G}, R)$ of genus $g$ is an embedding of the graph $\mathcal{G}$ into the orientable surface with $g$ handles. By $|V|,|E|$ and $|F|$, we denote the number of vertices, edges and faces of the given map (embedding). Note that $|V|-|E|+|F|=2-2 g$ by the Euler equation. For any regular map, let $k$ be the face-size and $n$ the valency of the underlying graph. Then one can easily see that $n|V|=k|F|=2|E|$. Consequently,

$$
g=1+\frac{1}{2}(|E|-|V|-|F|)=1+\frac{|V|}{2}\left(\frac{n}{2}-\frac{n}{k}-1\right) .
$$

Let $\mathcal{M}=\mathcal{M}(\mathcal{G}, R)$ be any regular map with underlying graph $\mathcal{G}$ of order $p q$ and of valency $n$. Then we have

$$
g=1+\frac{p q}{2}\left(\frac{n}{2}-\frac{n}{k}-1\right)
$$

Let $\operatorname{Mon}(\mathcal{M})=\langle R, L\rangle$ and $\operatorname{Aut}(\mathcal{M})=\langle r, \ell\rangle$. Then the mapping $\sigma: R \rightarrow r^{-1}$ and $L \rightarrow \ell^{-1}$ can be extended to an isomorphism from $\operatorname{Mon}(\mathcal{M})$ to $\operatorname{Aut}(\mathcal{M})$. By the definition of a regular orientable map, we have $k=|R L|$ and so $k=\left|r^{-1} \ell^{-1}\right|=|\ell r|$. Therefore in order to compute the genus of every regular map in Theorem 4.8, it is needed to determine $k=|\ell r|$ for each group $G_{i}$ for $1 \leq i \leq 6$.

Proposition 5.1 Let $\mathcal{M}$ be a regular map with simple underlying graph $\mathcal{G}$ of order pq for any two primes $p$ and $q$ (see Theorem 4.8) and let $g$ be the genus of $\mathcal{M}$.

(1) If $\mathcal{M} \cong \mathcal{M}_{1}(p, h, i)$, then $g=1+\frac{p(h-3)}{2}$.

(2) If $\mathcal{M} \cong \mathcal{M}_{2}(p, h, i)$, then $g=1+\frac{p(h-4)}{2}$ if $4 \mid h$, and $g=1+\frac{p(h-6)}{2}$ if $4 \nmid h$.

(3) If $\mathcal{M} \cong \mathcal{M}_{3}\left(p, q, t_{1}, t_{2}, i\right)$, then $g=1+\frac{p q(h-4)}{4}$ if $4 \mid h$, and $g=1+\frac{p q(h-6)}{4}$ if $4 \nmid h$.

(4) If $\mathcal{M} \cong \mathcal{M}_{4}(p, h, i)$, then $g=1+\frac{p^{2}(h-4)}{4}$ if $p=2$, or $p \geq 3$ and $4 \mid h$, and $g=1+\frac{p^{2}(h-6)}{4}$ if $p \geq 3$ and $4 \nmid h$.

(5) If $\mathcal{M} \cong \mathcal{M}_{5}(p)$, then $g=1+\frac{p(p-3)}{2}$.

(6) If $\mathcal{M} \cong \mathcal{M}_{6}(p, q, h, i, j)$, then $g=1+\frac{p}{2}(p q-2 p-q)$ if $p \neq q, g=1+\frac{p^{2}}{4}(h p-$ 4) if $p=q$ and $4 \mid h$, and $g=1+\frac{p^{2}}{4}(h p-6)$ if $p=q$ but $4 \nmid h$.

Proof: The proof is divided into six cases according to the six families $\mathcal{M}_{i}, 1 \leq i \leq 6$, of the maps in Theorem 4.8.

Case $1 \mathcal{M} \cong \mathcal{M}_{1}(p, h, i)$ : Let $G_{1}=G_{1}(p, h)$ with the admissible parameters in (4.1). Then $G_{1}=\langle x\rangle:\langle y\rangle \cong \mathbb{Z}_{p}: \mathbb{Z}_{2 h}$, where $h$ is odd. It follows from (4.7) that $q=2$, the valency $n=h$, and $r=y^{2 i}$ where $i \in \mathbb{Z}_{h}^{*}$ and $\ell=x y^{h}$. Then $\ell r=x y^{h} y^{2 i}=x^{i} y^{2 i+h}$. By $\langle x\rangle \triangleleft G_{1}$ it follows that $\left|y^{2 i+h}\right||| \ell r \mid$. Noting that $(2 i+h, 2 h)=1$, we have $\left|y^{2 i+h}\right|=2 h|k=| \ell r \mid$. Since every subgroup of order $2 h$ of $G_{1}$ is maximal, it follows $k=|\ell r|=2 h$. Inserting the values of $q, n$, and $k$ in (5.1), one can get (1). 
Case $2 \mathcal{M} \cong \mathcal{M}_{2}(p, h, i)$ : Let $G_{2}=G_{2}(p, h)$ with the admissible parameters in (4.2). Then $G_{2}=\langle x\rangle:\langle y\rangle \cong \mathbb{Z}_{p^{2}}: \mathbb{Z}_{h}$, where $h$ is even. It follows from (4.8) that $q=2$, the valency $n=h, r=y^{i}$ where $i \in \mathbb{Z}_{h}^{*}$ and $\ell=x y^{\frac{h}{2}}$. Then $\ell r=x y^{\frac{h}{2}} y^{i}=x y^{i+\frac{h}{2}}$. Note that $\left(i+\frac{h}{2}, h\right)$ is equal to 1 or 2 , according to whether $4 \mid h$ or $4 \mid h$, respectively. For a reason similar to Case 1 , we know that $k=|\ell r|=h$ or $\frac{h}{2}$, if $4 \mid h$ or $4 \nmid h$, respectively. Then the result in (2) follows from (5.1).

Case $3 \mathcal{M} \cong \mathcal{M}_{3}\left(p, q, t_{1}, t_{2}, i\right):$ Similar to Case 2 .

Case $4 \mathcal{M} \cong \mathcal{M}_{4}(p, h, i):$ Similar to Case 2 .

Case $5 \mathcal{M} \cong \mathcal{M}_{5}(p)$ : It is easy to see $k=|\ell r|=2 p$, as desired.

Case $6 \mathcal{M} \cong \mathcal{M}_{6}(p, q, h, i, j)$ : Let $G_{6}=G_{6}(p, q, h)$ with the admissible parameters given in (4.6). Then $G_{6}=\langle x\rangle:\langle y\rangle \cong \mathbb{Z}_{p^{2}}:\left(\mathbb{Z}_{q}: \mathbb{Z}_{h}\right)$.

First, assume $p \neq q$, so that $h=2$. In this case, it follows from (4.12) that $r=a^{\prime} y$ and $\ell=x^{i} y$, where $i \in \mathbb{Z}_{q}^{*} /\left(\mathbb{Z}_{q}^{*}\right)^{2+}$, and $a^{\prime}=t_{(1,0)}$ if $q \mid(p+1)$, and $a^{\prime}=t_{(1,1)}$ if $q \mid(p-1)$. Then $\ell r=x^{i} y a^{\prime} y$. Let $T$ be the translation subgroup of $\operatorname{AGL}(2, p)$. Since $y a^{\prime} y=\left(a^{\prime}\right)^{y} \in T \triangleleft \operatorname{AGL}(2, p)$ and $|x|=q$, it follows that $q|| \ell r \mid$. Since $x$ has no fixed points in its action on $V \backslash\{0\}$, the subgroup $T:\langle x\rangle$ cannot contain any element of order $p q$. Therefore $|\ell r|$ cannot be $p q$, and consequently, $k=|\ell r|=q$. Noting that $n=2 p$, the desired result in (6) is obtained by (5.1).

Next, assume $p=q$. In this case, it follows from (4.12) that $r=a^{\prime} y^{j}$ and $\ell=x^{i} y^{\frac{h}{2}}$, where $a^{\prime}=t_{(1,0)}, j \in \mathbb{Z}_{h}^{*}$, and $i \in \mathbb{Z}_{q}^{*} /\left(\mathbb{Z}^{*}\right)_{q}^{h+}$. Then $\ell r=x^{i} y^{\frac{h}{2}} a^{\prime} y^{j}=x^{i} y^{j+\frac{h}{2}}\left(a^{\prime}\right)^{y^{j}}$. Denote $x^{i} y^{j+\frac{h}{2}}$ by $y_{1}$. Then from (4.6) we know that $y_{1}=\left\|1,-i t^{j} ; 0,-t^{j}\right\|$. Therefore $\left|y_{1}\right|=h$ if $4 \mid h$, and $\left|y_{1}\right|=\frac{h}{2}$ if $4 \nmid h$. For a reason similar to the previous one, we get $\left|y_{1}\right||| \ell r \mid$. A direct computation shows that $1 \neq(\ell r)^{\left|y_{1}\right|} \in T$. Therefore $k=|\ell r|=p\left|y_{1}\right|$. Noting that $n=p h$, the desired result is obtained by inserting the values of $n$ and $k$ in (5.1).

\section{Acknowledgments}

The authors would like to acknowledge the support of $\mathrm{Com}^{2} \mathrm{MaC}-\mathrm{KOSEF}$; the first author would like to thank $\mathrm{Com}^{2} \mathrm{MaC}$ at POSTECH, Korea for its hospitality and to acknowledge the support of NSFC(19901022), BNSF(19920003), SYSF(19981002) and SRF for ROCS, SEM of China. The third author is partially supported by the Ministry of Education of the Slovak Republic, APVT-51-012502. Also, the authors are indebted to the referees who gave us many helpful suggestions.

\section{References}

1. N.L. Biggs, "Classification of complete maps on orientable surfaces," Rend. Mat. 4(6) (1971), 132-138.

2. N.L. Biggs and A.T. White, Permutation Groups and Combinatorial Structures, London Math. Soc. Lecture Notes 33, Cambridge University Press, Cambridge, 1979. 
3. C.Y. Chao, "On the classification of symmetric graphs with a prime number of vertices," Trans. Amer. Math. Soc. 158 (1971), 247-256.

4. L.E. Dickson, Linear Groups with an Exposition of the Galois Field Theory, Leipzig, 1901; Dover Publ. 1958.

5. S.F. Du, J.H. Kwak, and R. Nedela, "Regular embeddings of complete multipartite graphs," to appear in Europ. J. Combinatorics.

6. W. Feit and J.G. Thompson, "Solvability of groups of odd order," Pacific J. Math. 13 (1963), 775-1029.

7. A. Gardiner, R. Nedela, J. Širáň, and M. Škoviera, "Characterization of graphs which underlie regular maps on closed surfaces," J. London Math. Soc. 59 (1999), 100-108.

8. J.L. Gross and T.W. Tucker, Topological Graph Theory, Wiley, New York, 1987.

9. B. Huppert, Endliche Gruppen I, Springer-Verlag, Berlin, 1967.

10. B. Huppert and N. Blackburn, Finite Groups II, Springer-Verlag, New York, 1982.

11. L.D. James and G.A. Jones, "Regular orientable imbeddings of complete graphs," J. Combin. Theory Ser. B 39 (1985), 353-367.

12. G.A. Jones, "Maps on surfaces and Galois groups," Math. Slovaca 47 (1997), 1-33.

13. G. Jones and D. Singerman, "Theory of maps on orientable surfaces," Proc. London Math. Soc. 37 (1978), 273-307.

14. G. Jones and D. Singerman, "Belyi functions, hypermaps and Galois groups," Bull. London Math. Soc. 28 (1996), 561-590.

15. M.W. Liebeck and J. Saxl, "Primitive permutation groups containing an elements of large prime order," $J$. London Math. Soc. 31 (1985), 237-249.

16. P. Lorimer, "Vertex-transitive graphs: Symmetric graphs of prime valency," J. Graph Theory 8 (1984), 55-68.

17. D. Marušič and R. Scapellato, "Characterizing vertex-transitive $p q$-graphs with an imprimitive automorphism subgroup," J. Graph Theory 16 (1992), 375-387.

18. R. Nedela and M. Škoviera, "Exponents of orientable maps," Proc. London Math. Soc. 75(3) (1997), 1-31.

19. R. Nedela and M. Škoviera, "Regular maps of canonical double coverings of graphs," J. Combin. Theory Ser. B 67 (1996), 249-277.

20. C.E. Praeger, R.J. Wang, and M.Y. Xu, "Symmetric graphs of order a product of two distinct primes," J. Combin. Theory Ser. B $\mathbf{5 8}$ (1993), 299-318.

21. C.E. Praeger and M.Y. Xu, "Vertex primitive transitive graphs of order a product of two distinct primes," $J$. Combin. Theory Ser. B 59 (1993), 245-266.

22. R.J. Wang and M.Y. Xu, "A classification of symmetric graphs of order $3 p$," J. Combin. Theory Ser. B 58 (1993), 197-216.

23. M. Suzuki, Group Theory I, Springer- Verlag, New York, 1982.

24. M. Škoviera and J. Širán̆, "Regular maps from Cayley graphs. I: Balanced Cayley maps,” Discrete Math. 109 (1992), 265-276.

25. M. Škoviera and A. Zlatoš, "Construction of regular maps with multiple edges," in preparation.

26. S.E. Wilson, "Cantankerous maps and rotary embeddings of $K_{n}$," J. Combin. Theory Ser. B 47 (1989), $262-273$.

27. H. Wielandt, Finite Permutation Groups, Academic Press, New York, 1964. 